\title{
RELIABILITY OF PARENTAL SELF-REPORT OF INHALED CORTICOSTEROID ADHERENCE IN INNER-CITY MINORITY CHILDREN WITH PERSISTENT ASTHMA
}

\author{
M. Reznik, P.O. Ozuah
}

Pediatrics, Children's Hospital at Montefiore/Albert Einstein College of Medicine, Bronx, NY, USA

Background/aims: Physicians often rely on parental self-report of inhaled corticosteroid (ICS) adherence to guide asthma management. We examined reliability of parental self-report in assessing ICS adherence.

Methods: Prospective observational study of parents of young children with persistent asthma. At enrollment, children received a new, marked ICS with a dose counter. Parents were instructed to administer ICS 2 puffs twice daily. If used as directed, 120 actuations suffice for 30-days. Thirty days post-enrollment, we measured ICS adherence by parental self-report and objectively, using the number of puffs left on a dose counter. Parental self-reported ICS adherence was defined as: 100\%, administered ICS "every day"; $75 \%$, "almost every day ; 50\%, "several times a week"; $25 \%$, "once a week"; and $0 \%$, "less than once a week". Adherence was calculated as the number of puffs used relative to the number of puffs expected to have been used at 30-day follow-up. Wilcoxon signed-rank test compared the two adherence methods.

Results: 40 parents participated (mean age 32.7 (SD 6.6), 66\% Hispanic). Parental self-report overestimated ICS adherence ( $40 \%$ of parents reported being $100 \%$ adherent vs. 5\% being $100 \%$ adherent as per dose counter). Parents under-reported nonadherence (3\% reported $0 \%$ adherence vs. $10 \%$ having $0 \%$ adherence as per dose counter). Wilcoxon signed-rank test revealed a statistically significant overall difference between parental self-report and objectively measured adherence $(\mathrm{p}<.0001)$.

Conclusions: Parental self-report proved to be a non-reliable method for assessing ICS adherence. A dose counter that most ICS inhalers are equipped with may be a more reliable alternative measure. 